\section{Neue Therapieansätze bei der eosinophilen Ösophagitis}

Die eosinophile Ösophagitis ist weiterhin eine seltene, in den letzten Jahren aber doch häufiger zu beobachtende Erkrankung bei Kindern, die bisher diätetisch und z. T. systemisch, z. T. lokal mit Kortikoiden behandelt wird - mit allen damit verbundenen Problemen.

\begin{abstract}
$\Lambda$ us Tierversuchen weiß man inzwischen, dass Interleukin 5 ein wichtiger entzündungsfördernder Faktor bei der eosinophilen Ösophagitis ist. Wie beim M. Crohn wurde nun eine Substanz gesucht, die in diesen Prozess eingreift. Mittlerweile konnte ein neuer Wirkstoff aus der Gruppe der monoklonalen Antikörper synthetisiert werden, der Interleukin 5 hemmt bzw. neutralisiert: das Reslizumab.

Forscher aus den USA führten nun eine randomisierte, doppelblinde und placebokontrollierte Studie mit 226 jungen Patienten durch, die an einer eosinophilen Ösophagitis litten. Das Alter der Probanden betrug fünf bis 18 Jahre, ca. $90 \%$ hatten eine mäßige bis schwere Symptomatik wie etwa retrosternale
\end{abstract}

Schmerzen oder Schluckbeschwerden bzw. Erbrechen.

Spergel et al. teilten die klinischen Befunde mittels eines Scores, des "Physician's Eosinophilic Esophagitis Global Assessment", in fünf Schweregrade ein und zählten die intraepithelialen Eosinophilen in histologischen Präparaten der Speiseröhre. Die Patienten bekamen am Anfang, nach vier, acht und zwölf Wochen jeweils entweder 1, 2 oder 3 mg/kg KG Reslizumab oder aber Placebo als Infusion verabreicht.

Bei der Biopsie nach 15 Wochen war die Eosinophilen-Zahl bei den VerumPatienten im Vergleich zur Placebogruppe signifikant gesunken: um $59 \%$ in der Gruppe mit $1 \mathrm{mg}$, um $67 \%$ in der mit 2 und um $64 \%$ in der mit $3 \mathrm{mg}$. Auch kli- nisch war die Symptomatik im Verlauf gebessert: Zu Beginn hatten nur knapp $10 \%$ der Probanden keine oder kaum Beschwerden, jetzt waren es $56,4 \%$ in der 1-mg-Gruppe, 63,2\% in der 2-mg- bzw. $64,9 \%$ in der 3-mg-Gruppe. Aber zur Überraschung aller: In der PlaceboGruppe waren sogar $71,9 \%$ ohne wesentliche Beschwerden nach den knapp vier Monaten!

Spergel JM et al. Reslizumab in children and adolescents with eosinophilic esophagitis: results of a double-blind, randomized, placebocontrolled trial. J Allergy Clin Immunol 2012; 129: 456-63

Kommentar: In der Histologie wirkte Reslizumab bei der kindlichen eosinophilen Ösophagitis recht gut, verbesserte aber den klinischen Score gegenüber dem Spontanverlauf nicht. Ob sich im weiteren Verlauf die Rezidivrate damit aber senken lässt, was man sich ja infolge der Besserung der Eosinophilen-Zahl vorstellen könnte, wird nun in einer Anschluss-Studie überprüft. Als moderate (und bekannte) Nebenwirkungen der Therapie mit dem Präparat wurden Husten und Kopfschmerzen erwähnt. Dr. Ulrich Mutschler

\title{
Nussallergien natürlich behandeln
}

Frühere Untersuchungen mit einem Extrakt aus neun Kräutern der traditionellen chinesischen Medizin konnten zeigen, dass dieses Präparat Mäuse mit Erdnussallergie vor anaphylaktischen Reaktionen schützte. Inzwischen konnten auch erste Daten zur Wirkung bei einer kleinen Gruppe von Patienten mit Nussallergien erhoben werden.

\begin{abstract}
n einer offenen Studie bekamen 18 Probanden mit zuvor nachgewiesener Allergie gegen Erdnüsse und/oder Baumnüsse für ein halbes Jahr täglich $3 \times 6$ Tabletten $(=3 \times 3,3 \mathrm{~g})$ der Food Allergy Herbal Formula 2 (FAHF-2).
\end{abstract}

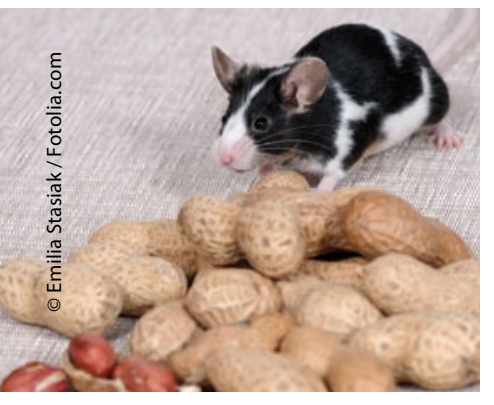

Im Mausversuch wurde ein Extrakt aus neun Kräutern erfolgreich gegen Nussallergie eingesetzt.
Die Ergebnisse dieser Pilotstudie waren ermutigend: Von 14 Patienten (im Alter von zwölf bis 27 Jahren), die der Studie letztendlich bis zum Schluss treu blieben, hatte keiner (bei üblicher Eliminationsdiät) eine Nuss-induzierte allergische Reaktion. Auch die Verträglichkeit war gut: Labor, EKG und Lungenfunktion blieben konstant im Normbereich. Die restlichen vier Patienten waren übrigens nicht wegen einer Unverträglichkeit ausgeschieden.

Funktionsanalysen des Immunsystems gaben auch wichtige Hinweise, dass dieser Kräuterextrakt - wie schon im Tierversuch - auch beim Menschen die Immunantwort auf Nuss-Allergene modifizieren dürfte: Provokationen mittels eines spezifischen Nuss-AllergenExtraktes induzierten am Ende der Studie bei den Probanden eine schwächere Aktivierung der Basophilen; und auch ihre Konzentration wie auch die der Eosinophilen im Differenzialblutbild sank von 5,3 auf $3 \%$.

Patil SP et al. Clinical safety of Food Allergy Herbal Formula-2 (FAHF-2) and inhibitory effect on basophils from patients with food allergy: extended phase I study. J Allergy Clin Immunol 2011; 128: 1259-65

Kommentar: Der chinesische Kräuterextrakt FAHF-2 erwies sich in der Verlängerung einer Phase-I-Studie als recht sicher und verträglich für junge Patienten mit Nussallergie. Unter der Behandlung ließen sich bei den Basophilen deren Aktivität und Anteil im peripheren Blut senken, ebenso ging die Zahl der Eosinophilen zurück. Diese Ergebnisse müssen jetzt natürlich in einer weiteren (Phase-II-) Studie und v. a. auch an einer größeren Zahl von Probanden überprüft werden.

Dr. Ulrich Mutschler 